\title{
DESENVOLVIMENTO DA QUÍMICA INORGÂNICA NO BRASIL
}

\author{
Henrique E. Toma* e Ana Maria da Costa Ferreira \\ Instituto de Química, Universidade de São Paulo, CP 26077, 05513-970 São Paulo - SP \\ Osvaldo A. Serra \\ Departamento de Química, Faculdade de Filosofia, Ciências e Letras de Ribeirão Preto, Universidade de São Paulo, \\ Av. Bandeirantes, 3900, 14049-901 Ribeirão Preto - SP
}

DEVELOPMENT OF INORGANIC CHEMISTRY IN BRAZIL. This article focuses on the development of Inorganic Chemistry in Brazil, including historical perspectives, scientific production (WEB-ISI data), international cooperation, teaching, literature and human resources, with particular emphasis on the last 25 years.

Keywords: inorganic chemistry; research in Brazil; scientific production; international cooperation.

\section{INTRODUÇÃO}

Compor um panorama da evolução da Química Inorgânica nos últimos 25 anos, foi uma incumbência difícil e arriscada, diante da complexidade da área. De fato, neste último quarto de século, a Química Inorgânica deixou de comportar a explosão de conhecimento gerado no contexto tradicional da química dos elementos, passando a incorporar também os avanços na química organometálica e metalorgânica, catálise, química bioinorgânica, materiais e, mais recentemente, na química supramolecular.

Apesar dos diferentes pontos de vista, a classificação histórica adotada na Química ainda vem sendo empregada na organização estrutural/administrativa/política, formalizada em termos das grandes áreas: inorgânica, orgânica, analítica e físico-química. É consenso que essa classificação, pelo seu caráter abrangente, é imprecisa ou inadequada, e assim, muitos advogam a necessidade de mudanças de paradigma na Química, como ciência. Realmente, é fácil enaltecer o rompimento das fronteiras, fictícias ou não, mirando para os horizontes da interdisciplinaridade. Porém, ao mesmo tempo é inegável a existência das especialidades ou divisões, permeando essas grandes áreas. Ao pensar em uma química sem fronteiras, podemos nos ver diante de um número ilimitado de especialidades, substituindo o que conhecemos atualmente como quatro grandes áreas. Essa aparente dicotomia, torna difícil traçar o perfil de desenvolvimento da Química Inorgânica nas últimas décadas, principalmente no Brasil. Avaliações sucessivas têm sido feitas, tomando como base ora a distribuição dos trabalhos em congressos nacionais ${ }^{1-4}$, como a SBPC, e mais recentemente a SBQ (onde a classificação é baseada nas Divisões Específicas da Organização), ora os eventos tradicionais ${ }^{5-7}$ que levam a conotação de Química Inorgânica, como o BMIC (antigo SNQI), ou ainda, os bancos de dados das Agências ${ }^{8-11}$ de Bolsas e Fomento (CAPES, CNPq) onde a área muitas vezes é definida pela classificação institucional do docente e, nem sempre, pela temática associada ao campo de atuação do pesquisador. Assim, tais avaliações nem sempre se concentram sobre o mesmo universo de pesquisadores ou de atividades. Algumas vezes, as atividades em materiais ou catálise são computadas dentro da área de Química Inorgânica. Por outro lado, é importante frisar que, com frequiência, a química de coordenação tem sido confundida com uma linha ou

\footnotetext{
*e-mail: henetoma@iq.usp.br
}

sub-área temática ${ }^{12}$, esquecendo-se os avaliadores de que o modelo de coordenação se aplica a todos os compostos que encerram núcleos metálicos em seu interior. Portanto, qualquer composto que contenha elementos metálicos em sua estrutura se enquadra na química de coordenação, mesmo que tenha função em catálise, conotações biológicas ou interesse na área de materiais. Da mesma forma, uma pesquisa que focalize compostos metal-orgânicos poderia ser classificada ao mesmo tempo como catálise, materiais, bioinorgânica, química teórica, etc. A citação que a pesquisa brasileira em Química Inorgânica tem sido restrita à química de coordenação não é pertinente, pois quase equivale a dizer que a nossa Química Orgânica tem sido limitada aos compostos de carbono. De fato, hoje, qualquer participante da reunião internacional de maior tradição na área de Inorgânica, conhecida como ICCC (International Conference on

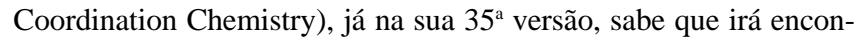
trar nesse evento as novidades mais recentes em bioinorgânica, catálise, espectroscopia, química supramolecular, materiais avançados e até nanotecnologia.

Assim, nesta apresentação, a Química Inorgânica foi tratada como grande área. Suas especialidades e focos de atividade foram destacados, como forma de delinear o desenvolvimento da área no país. Os números apresentados certamente não são absolutos, porém têm como base o banco universal de dados do ISI, através da Webofscience, e o banco de dados da CAPES e CNPq, onde o próprio pesquisador se classifica nas grandes áreas, ao preencher os formulários e cadastros pertinentes. Entretanto, mesmo considerando a relativa falta de homogeneidade nas informações, o uso de dados extraídos de avaliações passadas ainda se faz necessário, para tentar definir as tendências de desenvolvimento da grande área de Química Inorgânica no Brasil.

\section{UMA BREVE RETROSPECTIVA HISTÓRICA}

Ao contrário das outras áreas, na história da Química, a Inorgânica somente ganhou definição como tal há pouco mais de um século, como sendo a química voltada para a crescente classe de compostos não-orgânicos. A descoberta dos elementos e as tentativas de classificá-los racionalmente conduziram à formulação da Tabela Periódica, em 1869, por D. Mendeleev. A partir desse ponto, a curiosidade despertada a respeito da existência de novos elementos e a constatação das semelhanças entre suas propriedades atuaram como germe na nucleação da Química Inorgânica. Alfred Werner (18161919) foi o primeiro a questionar a validade das teorias e modelos 
aceitos na Química Orgânica, estruturando a nova Química Inorgânica em termos dos conceitos de coordenação e de estereoquímica. Curiosamente, após o enorme impacto da teoria de coordenação consagrada por Werner em sua grande obra (Neuere Anschauengen auf dem Gebiete der Anorganische Chemie, 1905), a Química Inorgânica passou por um período de menor atividade, que se estendeu até por volta de $1940^{13}$. Entretanto, de forma paradoxal, esse foi o período de maior renovação conceitual na história da ciência moderna, marcado pelo surgimento das teorias quânticas. Apesar da força do modelo estereoquímico de coordenação, introduzido por Werner desde o final do século XIX, a Química Inorgânica ainda não permitia um tratamento sistemático, a exemplo da química dos compostos orgânicos; muito menos exibia a lógica conceitual encontrada na FísicoQuímica. Dessa forma, os químicos inorgânicos, ao se ocuparem principalmente com a preparação e caracterização de diferentes compostos, acabaram gerando conhecimentos esparsos, embora importantes, porém ainda carentes de uma visão lógica ou sistemática mais desenvolvida. Por outro lado, sínteses, preparações e análises são processos interligados, e isso provavelmente explica o forte cunho analítico associado à área de Química Inorgânica, até a metade do século passado. Aos poucos, os modelos quânticos de ligação, como os introduzidos por H. Bethe (Teoria do Campo Cristalino) em 1929, e R. S. Mulliken (Teoria dos Orbitais Moleculares) em 1932, foram sendo assimilados pelos químicos inorgânicos. Os químicos aprenderam com Linus Pauling ${ }^{14}$ como os átomos se expressam através das ligações. Além da estereoquímica, o conhecimento das ligações dava um novo impulso ao estudo da Química Inorgânica.

Com a II Guerra Mundial o mundo ingressou na era atômica, marcada pelo descobrimento dos elementos transurânicos e pelos avanços na radioquímica. A disponibilização de isótopos permitiu a realização de experimentos importantes sobre o comportamento cinético e mecanístico dos compostos inorgânicos, o qual foi racionalizado por H. Taube, em 1949, com base nas teorias de ligação. O entendimento lógico do caráter lábil/inerte dos compostos de coordenação lançou a semente dos mecanismos de transferência de elétrons, propostos por Taube em 1953, definitivamente consagrados com o Prêmio Nobel que lhe foi outorgado em 1983.

No Brasil, as implicações da II Guerra Mundial tiveram reflexos imediatos na ciência, com a vinda de químicos de renome internacional, como Fritz Feigl ${ }^{15}$, Heinrich Rheinboldt ${ }^{16}$ e Heinrich Hauptmann ${ }^{17}$. As idéias renovadoras de Rheinboldt frutificaram na recém criada Universidade de São Paulo (1934) e o modelo de escola dirigida para a pesquisa acabou se estabelecendo no então Departamento de Química da Faculdade de Filosofia, Ciências e Letras. Feigl, Rheinboldt e Hauptmann impulsionaram o desenvolvimento da química no Brasil, especialmente nas áreas de química analítica, físico-química clássica e de química orgânica. Contribuíram para esse surto de desenvolvimento a fundação da Sociedade Brasileira para o Progresso da Ciência (SBPC) em 1948, do Conselho Nacional de Pesquisas (CNPq) em 1951 e da Coordenação de Aperfeiçoamento de Pessoal de Nível Superior (CAPES) nesse mesmo ano. Entretanto, nesse cenário de renovação, a Química Inorgânica no Brasil ainda vagava no compasso da química analítica ou descritiva, à espera do sopro renovador que viria mais tarde, trazido pelas teorias quânticas e de ligação. A necessidade de mudanças fez com que Ernesto Giesbrecht ${ }^{18}$, um dos discípulos de Rheinboldt, abandonasse sua trajetória científica na química de produtos naturais e biológica, partindo em 1956 para um estágio de pós-doutorado na Universidade de Illinois, na linha dos fosfatos de terras raras, onde trabalhou sob a supervisão do Prof. L. Audrieth ${ }^{19}$. Mais do que a realização de um trabalho científico, Giesbrecht trouxe, quando de seu retorno ao Brasil, a atualização científica que nortearia o ensino da Química Inorgânica na USP nos anos seguintes. Liderou inúmeras iniciativas de renovação do ensino da química, catalisando a introdução de programas, como o CBA (Chemical
Bond Approach) e redirecionando o ensino descritivo da Química Inorgânica, para um enfoque centrado nas relações periódicas e nas teorias de ligação. Nesse período, juntamente com G. Vicentini e M. Perrier, foram implantadas as primeiras linhas de pesquisa focalizando a química dos compostos de Terras Raras no Brasil. Paralelamente, P. Krumholz ${ }^{20}$, ex-discípulo de Feigl, que se encontrava no Brasil desde 1941, além de desenvolver processos de extração e separação de Terras Raras na antiga Orquima S.A., dedicava-se também à espectroscopia de absorção desses elementos ${ }^{21,22}$.

No contexto universal, as idéias consagradas por Pauling, em termos da natureza das ligações químicas, possibilitaram um avanço expressivo na sistemática dos compostos inorgânicos, principalmente na visão estrutural. No Brasil, a química das Terras Raras teve notável desenvolvimento desde a sua implantação, tornando-se a principal especialidade de pesquisa em Inorgânica no país, até os anos 80 . Se, por um lado, o desenvolvimento da química das Terras Raras foi decisivo para o andamento do programa de pós-graduação e formação de doutores na área, a assimetria gerada já era visível no final dos anos 60, delineando-se um quadro de carência de pesquisas em outras especialidades da Química Inorgânica; particularmente em temas envolvendo os metais de transição. Nesse período, apesar da existência de alguns grupos de renome internacional, como o de P. Krumholz na área de complexos diimínicos de ferro e metalocarbonilos, a pequena diversidade de pesquisas na área de Inorgânica tornou-se bastante preocupante. Ao lado das turbulências políticas, o país via-se diante da expectativa de uma nova onda de desenvolvimento, marcada pela instalação dos pólos petroquímicos e pela revolução industrial. Catálise e materiais tornaram-se alvo de crescente interesse. Nesse cenário, visando a expansão e a diversificação das atividades de pesquisa em Química, várias iniciativas de cooperação internacional foram implementadas, como o Programa Multinacional de Química, apoiado pela Organização dos Estados Americanos (OEA) e o Programa de Cooperação entre a National Academy of Sciences (NAS) e o CNPq (1969/1975). Particularmente na área de Química Inorgânica, o impacto desses programas foi marcante, com envolvimento de pesquisadores como Larry Thompson (1969), John Malin (1969-72) e Henry Taube (1969-75) em São Paulo, e Harry Gray (1969-72) no Rio de Janeiro, e o início de pesquisas na área de reatividade química, utilizando técnicas de cinética rápida e espectroscopia. Sob a supervisão de Henry Taube, foi implantado o laboratório de cinética e mecanismos de reações inorgânicas no Instituto de Química da USP. Nesse laboratório foram iniciadas novas linhas de pesquisa focalizando a química de aminas de rutênio e ósmio, ainda bastante incipiente, as quais viriam a se expandir para a química dos cianoferratos, completando a tríade dos elementos. A influência de Taube na Química Inorgânica brasileira não ficou limitada à sua atuação pessoal, tendo sido principalmente exercida através de interações com pesquisadores formados em sua Escola, em âmbito nacional e internacional, a partir dos anos 70. Esse fato explica a elevada densidade de trabalhos sobre a química do rutênio e dos cianoferratos em nosso país, até os tempos atuais.

Outro evento marcante nos anos 70 foi o Seminário Latino-Americano de Química (SELAQ - 1972), que permitiu a interação da comunidade com pesquisadores de grande expressão, como J. C. Bailar, O. Fischer, S. Kirschner, E. Fluck e V. Gutman. As atividades do período, concentradas na Universidade de São Paulo, ao lado de iniciativas de formação e captação de doutores no exterior, como na UFMG, UnB, UFSC e UNICAMP, foram decisivas para a formação das lideranças em Química Inorgânica, que promoveram enorme diversificação e expansão da área, nas três últimas décadas.

Em 1974 foi publicado o Plano Básico de Desenvolvimento Científico e Tecnológico (PBDCT), juntamente com o Plano Nacional de Pós-Graduação, estabelecendo metas importantes para a consoli- 
dação da pesquisa e pós-graduação no país. Esse período ainda foi marcado pela fundação da Sociedade Brasileira de Química, em 1977. Merecem destaque a criação do Programa Nacional de Química (Pronaq, 1979), e a implementação do Programa de Apoio ao Desenvolvimento Científico e Tecnológico ${ }^{23-24}$ (PADCT, 1984) financiado pelo Banco Mundial, com várias renovações até a presente data. Ainda no final da década de 90 foi lançado o Programa de Núcleos de Excelência (Pronex) promovido pelo Ministério de Ciência e Tecnologia e, já no novo século, o Programa dos Institutos do Milênio $(\mathrm{CNPq})$.

No Estado de São Paulo, as iniciativas da FAPESP foram decisivas para a manutenção da infraestrutura de pesquisa das Universidades, além de proporcionar um apoio constante ao fomento de balcão e aos projetos temáticos, dando um impulso bastante significativo ao desenvolvimento de pesquisa em todas as áreas. A criação das FAPs em outros Estados também deve ser destacada, lamentando-se porém a sua ausência nas regiões de menor desenvolvimento e mais carentes de recursos para pesquisa.

\section{REUNIÕES DA ÁREA DE QUÍMICA INORGÂNICA}

O I Simpósio Nacional de Química Inorgânica (I SNQI) foi realizado em 1982, nas dependências do Instituto de Química da UNESP, em Araraquara. Nesse evento foi feita uma avaliação da área, conforme relatado por A. C. Massabni ${ }^{5}$, baseado em um questionário distribuído aos participantes. Essa atividade tornou-se tradicional dentro das reuniões da área de Química Inorgânica. Em 1984, o II SNQI foi realizado em São Paulo, coincidindo com a reunião anual da SBPC, tendo como tema central o cinqüentenário da Universidade de São Paulo. Em 1986, o III SNQI foi realizado em Campinas, ainda visando um mapeamento dos grupos atuantes na área. O IV SNQI foi realizado em 1988, nas dependências da PUC $-\mathrm{RJ}^{6}$ e já mostrava a pujança das pesquisas neste campo. A partir de 1990, as reuniões da SBQ foram organizadas de forma independente, separando-se das reuniões da SBPC, tendo como local a cidade de Caxambu - MG. Embora ainda sem o reconhecimento oficial como Divisão de Inorgânica os participantes dos SNQIs decidiram que as reuniões seriam realizadas em conjunto, em parte como forma de apoio à Reunião da SBQ, e também pela conveniência de contar com infraestrutura e suporte financeiro. Mesmo inserido na Reunião Anual da SBQ, o SNQI continuou mantendo sua identidade, organizando o Programa Científico na área, incluindo a escolha dos conferencistas e convidados, além do tradicional jantar de confraternização. Dessa forma, os V, VI, VII e VIII SNQIs foram realizados em 1990, 1992, 1994 e 1996, em Caxambu. Em 1994 foi oficializada a criação da Divisão de Química Inorgânica na SBQ (vide Apêndice). A partir de 1998, seguindo o exemplo das outras áreas, o SNQI voltou a se organizar de forma independente, buscando entretanto um espaço internacional, com a sigla BMIC (Brazilian Meeting on Inorganic Chemistry). O IX BMIC teve lugar na cidade de Angra dos Reis, em 1998. O sucesso alcançado foi repetido em 2000, no $X$ BMIC realizado no Costão do Santinho, em Santa Catarina. O XI BMIC deverá ocorrer em 2002, na cidade de Ouro Preto, Minas Gerais, incluindo também participantes internacionais.

\section{PRODUÇÃO CIENTÍFICA EM QUÍMICA INORGÂNICA}

Com base em um levantamento da produção científica dos pesquisadores brasileiros atuantes em Química Inorgânica, nos últimos 25 anos, procurou-se verificar além da quantidade de trabalhos realizados, quais os temas mais investigados, a contribuição específica das diferentes instituições no país, as parcerias internacionais, as revistas mais utilizadas para divulgação dos trabalhos; enfim, traçar um perfil do que vem ocorrendo nesta área de pesquisa, em nosso país. O banco atual de dados do Institute of Scientific Information, ISI, se estende desde 1945 até o presente; contudo, algumas revistas atualmente indexadas, como Química Nova e os Anais da Academia Brasileira de Ciências, deixaram de ser incluídas por alguns períodos no passado, principalmente na década de 80 .

Na compilação dos dados foram utilizados alguns critérios e/ou metodologias. Inicialmente, todos os pesquisadores (docentes) cujos nomes constassem de pelo menos um trabalho, na década de 90, nos Resumos das Reuniões Anuais da SBQ e/ou dos SNQI/BMIC foram selecionados, num total de aproximadamente 700 autores. Estes autores tiveram sua produção científica pesquisada no WEB-ISI, restringindo-se o endereço a "Brazil", isto é, considerando-se apenas os trabalhos em que o local de origem do pesquisador brasileiro indicado no trabalho fosse o Brasil. Alguns nomes exigiram uma pesquisa mais elaborada; como aqueles que incluem "de", "dos", "Filho", etc, visto que podem aparecer de diferentes formas nos trabalhos. Foram assim obtidos 4392 publicações envolvendo pesquisadores brasileiros, no período de 1977 a 2001, os quais foram armazenados, usando o programa ENDNOTE, e passaram por uma seleção, para serem reconhecidos como trabalhos inorgânicos. Nessa seleção, foram consideradas as revistas, os autores e os temas enfocados. Contribuições de autores reconhecidamente inorgânicos, mas cujo trabalho não o era, foram excluídos; por outro lado, trabalhos tipicamente inorgânicos, embora de autores não tradicionalmente inorgânicos, foram considerados, visto que o objetivo era produzir um levantamento da contribuição brasileira para o desenvolvimento da Química Inorgânica. Usando-se esses critérios, chegou-se a 2273 publicações, as quais foram então analisadas quanto aos sub-temas, locais, instituições, palavras-chave, etc.

Possivelmente os números obtidos não contemplam toda a produção da área, dada a complexidade da mesma. Entretanto, dados mais confiáveis sobre a produção das áreas nas décadas passadas não existem ou não foram disponibilizados pelas Agências competentes. Dessa forma, deve ficar claro que os dados produzidos neste trabalho oferecem apenas o valor mínimo, basal, da produtividade acadêmica, estando excluídos os trabalhos em revistas não indexadas no ISI, bem como aqueles não capturados através do procedimento descrito. Se os números não são absolutos, com certeza as tendências são válidas e bastante reveladoras. De fato, como mostrado na Tabela 1, a evolução das publicações sofreu um aumento abrupto a partir de 1990, rompendo o patamar de 30 publicações para um crescimento exponencial, multiplicando-se por um fator superior a 10 vezes, em apenas uma década. De um total de 2273 publicações indexadas, no período 1977 a 2001, apenas $17,9 \%$ correspondem ao período 1977-1990, com 82,1\% sendo produzidos a partir de 1991 . Se levarmos em conta os últimos 5 anos, a produção neste período corresponde a 55,0\% do total (1250 artigos).

Esse vertiginoso aumento reflete uma série de fatores, entre os quais, o impacto do PADCT; dos processos de avaliação acadêmica, como o da CAPES; do aumento da competitividade associado às bolsas de produtividade; e também da consolidação da Sociedade Brasileira de Química. Note-se que a época em questão coincide com o início da realização das Reuniões Anuais da SBQ, em separado da SBPC. A maior interação entre os pesquisadores e a valorização dos trabalhos promovida pela SBQ, dando-lhes uma projeção nacional e internacional, pode ter sido um fator importante nesta aceleração observada. Isso está claramente refletido na duplicação do número de trabalhos anuais logo no início dos anos 90, e depois a cada 3 ou 4 anos.

As revistas mais freqüentemente utilizadas para a publicação dos trabalhos estão destacadas na Tabela 2. Cerca de $67 \%$ do total de trabalhos se concentram em 35 revistas. Note-se que cerca de $8,2 \%$ 
Tabela 1. Produção Científica na Área de Química Inorgânica (Fonte WebofScience) no período de 1977 a 2001

\begin{tabular}{lclc}
\hline Ano & Artigos Indexados & Ano & Artigos Indexados \\
\hline 1977 & 25 & 1990 & 40 \\
1978 & 20 & 1991 & 58 \\
1979 & 20 & 1992 & 72 \\
1980 & 30 & 1993 & 95 \\
1981 & 34 & 1994 & 103 \\
1982 & 34 & 1995 & 135 \\
1983 & 23 & 1996 & 152 \\
1984 & 19 & 1997 & 193 \\
1985 & 29 & 1998 & 174 \\
1986 & 28 & 1999 & 261 \\
1987 & 37 & 2000 & 340 \\
1988 & 31 & 2001 & $>282$ \\
1989 & 38 & Total: & $\mathbf{2 2 7 3}$ \\
\hline
\end{tabular}

de todos os trabalhos foram publicados nas revistas brasileiras da área de Química, Química Nova e Journal of Brazilian Chemical Society, com índices de impacto i $=0,399$ e 0,402, respectivamente; e 2,9\% dos trabalhos foram publicados nos Anais da Academia Brasileira de Ciência. Esse número está abaixo do real, visto que a revista foi reformulada visando maior inserção internacional a partir de 2000, voltando a ser indexada pelo ISI. As Academias de Ciência em todos os países refletem os padrões nacionais de excelência, e as mudanças de paradigma dos Anais representam iniciativas importantes, que deverão ter impacto positivo nos próximos anos.

É notório que parte da produção está sendo direcionada para revistas internacionais em diferentes e importantes sub-áreas, como materiais $(5,9 \%)$, bioinorgânica $(1,8 \%)$, catálise $(2,3 \%)$, organometálicos $(1,8 \%)$, eletroquímica $(1,5 \%)$ e termoquímica $(6,6 \%)$. Estes números são bastante aproximados, já que foram considerados somente os artigos nas revistas especializadas nestes temas. Assim, como exemplos, a bioinorgânica está sendo medida apenas pelos artigos publicados em Journal of Inorganic Biochemistry; e catálise, por aqueles artigos publicados em Applied Catalysis A e Journal of Molecular Catalysis. As revistas em Química Inorgânica com maior índice de impacto, Inorganic Chemistry $(\mathrm{i}=2,7)$ e Journal of Chemical Society, Dalton Transactions $(i=2,6)$ receberam 4,9\% de nossa produção total nestes 25 anos. Revistas tradicionais e típicas da área de Inorgânica (Inorganica Chimica Acta, Polyhedron, Journal of Coordination Chemistry, Transition Metal Chemistry, Z. Anorg. Allg. Chem. e J. Alloy Compd.) que valorizam a síntese, caracterização e estudos de reatividade de compostos inorgânicos publicaram $16,9 \%$ de nossos trabalhos.

Os restantes 33,0\% da produção estão disseminados em uma ampla gama de diferentes revistas, incluindo também áreas afins, como físico-química, analítica ou bioquímica, evidenciando a interdisciplinaridade dos estudos desenvolvidos pela comunidade inorgânica.

Através destes dados pode-se perceber a grande diversidade de investigações realizadas, voltadas para os diferentes aspectos da Química Inorgânica. Trabalhos mais conceituais, pesquisas centradas em medidas espectroscópicas, estudos cinéticos, eletroquímicos e termoquímicos, diferentes aplicações de compostos inorgânicos, tanto em sub-áreas mais tradicionais como catálise ou organometálicos, como em temas mais atuais como bioinorgânica, materiais ou sistemas supramoleculares, todos têm sido abordados pelos pesquisadores nacionais. Este quadro em nível nacional reflete também o que vem ocorrendo em nível internacional, com diversificação enorme dos assuntos focalizados pela comunidade inorgânica.

\section{PRODUÇÃO CIENTÍFICA NAS INSTITUIÇÕES UNIVERSITÁRIAS}

A Tabela 3 proporciona uma visão comparativa dos números de artigos indexados em Química Inorgânica, produzida pelas principais instituições universitárias brasileiras, nos últimos 25 anos

As universidades federais participam de $36 \%$ da produção científica em Química Inorgânica, ao passo que as universidades estadu-

Tabela 2. Principais Revistas Utilizadas por Pesquisadores Brasileiros Atuantes na Área de Química Inorgânica, no período de 1977 a 2001

\begin{tabular}{lclr}
\hline Revista & No. artigos & Revista & No. Artigos \\
\hline Acta Crystallogr. C & 44 & J. Mater. Res. & 9 \\
An. Acad. Bras. Ci. & 66 & J. Mater. Sci. & 13 \\
Appl. Catal. A - Gen. & 12 & J. Mater. Sci. Lett. & 15 \\
Eclet. Quim. & 14 & J. Mol. Catal. A & 41 \\
Electrochim. Acta & 18 & J. Mol. Struct. & 22 \\
Hyperfine Interact. & 16 & J. Non-Cryst. Solids & 77 \\
Inorg. Chem. & 65 & J. Organomet. Chem. & 25 \\
Inorg. Chim. Acta & 100 & J. Photochem. Photobiol. A & 18 \\
J. Alloys and Compd. & 90 & J. Sol-Gel Sci.Technology & 9 \\
J. Braz. Chem. Soc. & 102 & J. Therm. Anal. & 35 \\
J. Chem. Educ. & 10 & Langmuir & 17 \\
J. Chem. Res. - S & 21 & Organometallics & 15 \\
J. Chem. Soc., Chem. Commun. & 22 & Polyhedron & 91 \\
J. Chem. Soc., Dalton Trans. & 47 & Quim. Nova & 84 \\
J. Colloid Interf. Sci. & 39 & Spectrochim. Acta, Part A & 16 \\
J. Coord. Chem. & 43 & Spectroscopy Lett. & 20 \\
J. Electroanal. Chem. & 16 & Thermochim. Acta & 115 \\
J. Inorg. Biochem. & 41 & Transit. Metal Chem. & 30 \\
J. Inorg. \& Nuclear Chem. & 23 & Z. Anorg. Allg. Chem. & \\
J. Luminescence & 23 & & \\
J. Less-Common Metals & 9 & Total: & \\
J. Mater. Chem. & 20 & & \\
\hline
\end{tabular}


ais paulistas, de 53\%. Em termos regionais, o Estado de São Paulo foi responsável por $56 \%$ da produção científica em Química Inorgânica, no último quarto de século, seguido do Rio Grande do Sul, 6,2\% e Minas Gerais, 6\%.

Tabela 3. Produção Científica em Química Inorgânica, das Principais Instituições Universitárias, no Período 1977/2001

\begin{tabular}{lcr}
\hline Universidade & Número de Artigos & $\%$ \\
\hline UFMS & 12 & 0,5 \\
UFCG & 16 & 0,7 \\
UFBA & 12 & 0,5 \\
UFCE & 25 & 1,1 \\
UFGO & 13 & 0,5 \\
UFMG & 120 & 5,3 \\
UFOP & 17 & 0,7 \\
UFPB & 48 & 2,1 \\
UFPE & 78 & 3,4 \\
UFPR & 73 & 3,2 \\
UFRN & 35 & 1,5 \\
UFRS & 98 & 4,3 \\
UFRJ & 55 & 2,4 \\
UFSC & 83 & 3,6 \\
UFSM & 44 & 1,9 \\
UNB & 24 & 1,1 \\
UNESP & 197 & 8,7 \\
UNICAMP & 386 & 17,0 \\
UFSCAR & 67 & 2,9 \\
USP/SC & 851 & 3,7 \\
USP/RP & 293 & 12,9 \\
USP/SP & &
\end{tabular}

\section{COOPERAÇÃO INTERNACIONAL}

Cerca de $20 \%$ do total de trabalhos contaram com a colaboração de pesquisadores internacionais, provavelmente referentes a programas de Pós-Doutorado ou Doutoramento no exterior, conforme mostrado na Tabela 4. A maior interação ocorreu com USA $(3,5 \%)$ e França (3,3\%), seguidos de Alemanha (2,9\%), Espanha (2,6\%), Inglaterra $(1,6 \%)$, Itália $(1,5 \%)$ e Canadá $(1,3 \%)$. Os demais apareceram individualmente em menos de $1 \%$ da produção total.
Tabela 4. Principais colaborações internacionais

\begin{tabular}{lc}
\hline País & No. artigos \\
\hline USA & 80 \\
França & 74 \\
Alemanha & 66 \\
Espanha & 59 \\
Inglaterra & 36 \\
Itália & 34 \\
Canadá & 29 \\
Argentina & 20 \\
Portugal & 19 \\
Chile & 12 \\
Países Baixos & 9 \\
Venezuela & 9 \\
Rússia & 9 \\
Irlanda & 6 \\
Suíça & 5 \\
Uruguai & 3 \\
outros & 10 \\
\hline Total: & 480 \\
\hline
\end{tabular}

\section{LINHAS TEMÁTICAS}

Além das revistas mais utilizadas, procurou-se também identificar quais as temáticas mais abordadas por nossos pesquisadores. Diferentes palavras-chaves foram então testadas, como classificatórias das investigações brasileiras. Na Tabela 5 estão listadas algumas das mais adequadas, como indicativas dos interesses de nossos pesquisadores e os correspondentes artigos em que foram selecionadas.

Catálise aparece agora em $6,7 \%$ do total publicado, eletroquímica em $9,8 \%$, novos materiais em $14,4 \%$, termoquímica em $14,3 \%$ e organometálicos em 3,7\%. A Química do rutênio comparece em 9\% do total de 2273 artigos, lantanídios em 10,4\%, porfirinas em 4,2\% e luminescência em $8,6 \%$. A diferença observada ao se comparar os dados obtidos entre as revistas mais utilizadas e estes, a partir das palavras-chaves, indica que nem sempre as palavras-chaves estão sendo coerentemente escolhidas. Por exemplo as palavras-chaves bioinorganic, mimic or mimetic foram utilizadas em apenas 16 dos artigos, enquanto o número de artigos publicados só no Jounal of Inorganic Biochemistry, revista voltada para estudos bioinorgânicos,

Tabela 5. Palavras-Chave mais utilizadas em artigos de pesquisadores brasileiros atuantes na área de Química Inorgânica

\begin{tabular}{lclr}
\hline Palavras-Chave & No. artigos & Palavras-Chave & No. Artigos \\
\hline catalysis / catalytic activity & 152 & nitrosyl & 25 \\
bioinorganic / mimic / mimetic & 16 & organometallic & 84 \\
cobalt & 94 & platinum & 67 \\
chromium & 38 & porphyrin & 96 \\
copper & 174 & rare earth / lanthanides & 236 \\
electrochemical / electrochemistry & 223 & rhodium & 33 \\
europium & 109 & ruthenium & 205 \\
glasses & 111 & sol / gel & 221 \\
hydrogen peroxide & 27 & supramolecular / devices & 43 \\
iron & 292 & thermal / thermochemistry & 325 \\
luminescence & 196 & tin & 98 \\
manganese & 41 & titanium & 88 \\
materials / new materials & 328 & vanadium & 61 \\
molecular sieves & 12 & zeolite / zeolites & 45 \\
nanocomposites & 21 & zinc & 100 \\
nickel & 109 & & \\
\hline
\end{tabular}


foi de 41. Assuntos como compostos de nitrosila compareceram em $1,1 \%$ das publicações (25), usando nitrosyl como palavra-chave. Nanocompósitos apareceram em 0,9\% (21) e vidros em 5,0\% (111) dos artigos publicados.

\section{LITERATURA E ENSINO DE QUÍMICA INORGÂNICA}

Como a evolução da pesquisa tem vínculos com a literatura acadêmica e o ensino, nos pareceu oportuno traçar um paralelo histórico, destacando alguns textos mais antigos, encontrados na Biblioteca Principal de Química (USP). Nesse contexto, um livro que chamou nossa atenção, foi o de J. M. Teixeira ${ }^{25}$ (Lente catedrático da Faculdade de Medicina do Rio de Janeiro) publicado em 1878, com o título Noções de Química Inorgânica. Esse texto tratava a Química Inorgânica em termos de duas grandes categorias, a dos metalóides e a dos metais, apresentando aspectos preparativos, propriedades químicas, aplicações e aspectos toxicológicos. Ainda sem adotar a classificação formal da tabela periódica de Mendeleev, o agrupamento dos elementos era feito pela valência: elementos monovalentes $(\mathrm{F}$, $\mathrm{Cl}, \mathrm{Br}, \mathrm{I})$, bivalentes $(\mathrm{O}, \mathrm{S}, \mathrm{Se}, \mathrm{Te})$, trivalentes $(\mathrm{B})$, tetravalentes $(\mathrm{C}$, $\mathrm{Si}$ ) e pentavalentes (N, P, As, Sb). Esse tipo de enfoque deve ter sido universal, pois também é encontrado no livro Grunlinien der Anorganischen Chemie publicado na Alemanha, em 1912, por W. Ostwald $^{26}$. Outro texto importante, escrito por E. H. Riesenfeld ${ }^{27} \mathrm{em}$ 1928, foi voltado para o ensino da Química Analítica Inorgânica, ainda hoje ministrado sob a denominação de Química Analítica Qualitativa em muitas Escolas.

Os livros escritos por C. Oppenheimer ${ }^{28}$, em 1920, e por K. A. Hofmann $^{29}$, em 1924, além de apresentarem uma visão descritiva da química dos não metais, metais e das substâncias radioativas, introduziram como novidade o modelo atômico proposto por Bohr. Embora ainda não delapidada pela mecânica quântica, essa iniciativa já sinalizava a mudança de paradigma que estava por vir, no ensino da Química Inorgânica. Tal mudança é bastante nítida no livro de H. J. Emeléus e J. S. Anderson ${ }^{30}$, publicado em 1938, onde a Química Inorgânica passou a ser enfocada conceitualmente, através da teoria atômica, relações periódicas, estruturas e propriedades magnéticas, assumindo maior abrangência no trato dos compostos de coordenação, compostos de elementos não metálicos, radicais e espécies ativas de oxigênio, metalocarbonilos, compostos intermetálicos, sólidos inorgânicos, compostos não estequiométricos, solventes não aquosos e radioquímica. A preocupação com os aspectos conceituais atingiu o seu ápice com L. Pauling ${ }^{13}$ em 1940, quando racionalizou o comportamento químico e a estrutura, a partir dos fundamentos que regem a natureza da ligação química. Esse enfoque deu novo sentido à química estrutural, como pode ser visto na primeira edição do texto consagrado de A. F. Wells ${ }^{31}$, que vem sendo reeditado, com atualizações sucessivas, até o presente. Esses textos tiveram enorme influência no estilo didático de autores consagrados na década de 50, como nos textos de N. V. Sidgwick ${ }^{32}$ (1950), T. Moeller ${ }^{33}$ (1952), E. S. Gilreath ${ }^{34}$ (1958), e na década de 60 , como os de J. Kleinberg, W. J. Argersinger Jr. e E. Griswold ${ }^{35}$ (1960), F. A. Cotton e G. Wilkinson $^{36}$ (1962), e C. S. G. Phillips e R. J. P. Williams ${ }^{37}$ (1965). Um texto, em especial, publicado por L. G. Orgel ${ }^{38}$ em 1960, abordou com destaque, a química dos metais de transição baseada na teoria do campo ligante e conceitos de simetria. A partir dessa inovação, observa-se uma tendência de separação conceitual da química dos elementos representativos, como o texto de W. Jolly ${ }^{39}$ (1966), da química dos elementos de transição, esta última sempre enfocada em termos da teoria do campo ligante. A abordagem conceitual baseada em teoria de grupo foi a seguir tornada bastante didática por $\mathrm{F}$. A. Cotton ${ }^{40}$ (1963), exercendo profundas influências no ensino da química inorgânica a partir dos anos 60 .
No Brasil, o texto de Química Inorgânica publicado por O. A. Ohlweiler ${ }^{41}$, em 1971, tornou acessível um volume imenso de conhecimento descritivo na área. Gradualmente, porém, o ensino da Química Inorgânica baseada em teoria de grupo e de campo ligante acabou crescendo, tanto no país como no exterior, atingindo uma tal dimensão a ponto de suscitar críticas, em termos da carência de conteúdo descritivo, que levava à desinformação sobre aspectos básicos da natureza e propriedades dos compostos. As duas tendências pedagógicas (descritiva e conceitual) acabaram convergindo já no final dos anos 70 para o esquema atual, onde se faz o desenvolvimento de aspectos conceituais (estrutura, ligação, termodinâmica), buscando a racionalização do conteúdo descritivo na química dos elementos, e a compreensão das propriedades, mecanismos, catálise, e química organometálica. Nas últimas décadas, os textos passaram a incorporar capítulos dedicados à química bioinorgânica e à química dos materiais. Em muitas Universidades, como a USP, a química de coordenação passou a ser ensinada como disciplina destacada da inorgânica básica, incorporando conceitos e enfoque físico-químicos, e interligando aspectos analíticos, biológicos, de catálise e de materiais. A grande diversidade de autores modernos está sendo omitida deste artigo, pois fazem parte da literatura didática corrente em química inorgânica. Entretanto, não podemos deixar de comentar, que salvo alguns $\operatorname{casos}^{42-43}$, a participação de autores brasileiros na literatura didática, em Química Inorgânica, ainda permanece inexpressiva.

\section{RECURSOS HUMANOS}

Nos levantamentos recentes feitos pela CAPES, o número total de docentes em Química evoluiu de pouco mais de uma centena de doutores em $1970^{44}$, para a faixa dos 300 a 500 docentes nos anos $80^{45}$, até o patamar de 900 docentes na década de $90^{46}$, permanecendo praticamente inalterado desde então ${ }^{47}$. Os indicadores revelam que mesmo com o congelamento no quadro de docentes das Universidades Públicas na última década, a produção científica global, de química, aumentou significativamente, em termos qualitativos e quantitativos, passando de 477 publicações no biênio 83/84, para 836 no biênio 90/91, 1870 no biênio 96/97 e 4174 no triênio 98/2000 Nesse período, a preferência por publicações em revistas estrangeiras, em relação às nacionais, cresceu sistematicamente, de $67 \%$ no biênio $83 / 84$ para $85 \%$ no triênio $98 / 2000$.

Uma outra visão que reflete esse progresso é dada pela variação do perfil de distribuição dos bolsistas de produtividade do CNPq ao longo dos tempos. No início dos anos 80, a Química tinha cerca de 276 bolsistas de produtividade distribuída nos níveis I, II e III ${ }^{44,45}$. A área de Química Inorgânica contribuía com 16\% nesse universo de bolsistas, comparado com 8 (QA), 42 (QO) e 34\% (FQ) para as demais áreas (Tabela 4). No início dos anos 90, a bolsa nível III já não era mais implementada. O número total de 333 bolsas sofreu pouca alteração ao longo da década, flutuando com altos e baixos, entre 327 e 353 bolsas. O percentual de atendimento qualificado era da ordem de $80 \%$ no início dos anos 90 , caindo gradualmente para $35 \%$ em 1997 e recuperando-se parcialmente nos anos seguintes, até o índice atual de $50 \%$. Note-se que esse índice não representa a probabilidade de atendimento para um pesquisador ingressante no sistema. A disponibilidade real do número de bolsas para novos pretendentes é extremamente baixa, visto que o índice de renovação no nível I é pequeno, em função do perfil diferencial, bastante destacado, dos pesquisadores, à medida em que evoluem até o topo da classificação. Nos níveis IIC e IIB, onde a renovação é mais intensa, o ingresso de um novo bolsista no sistema é praticamente condicionado à retirada de outro, de menor produtividade, dada a constância no número total de bolsas. Isso está tornando cada vez mais difícil a 
concessão de bolsas para pesquisadores jovens, em todas as áreas, pressionando por outro lado, um maior desempenho por parte de todos os bolsistas. De fato, apesar dos efeitos perversos da restrição do número de bolsas de pesquisa, nota-se em todos os indicadores de produção, um melhor desempenho dos bolsistas, tanto qualitativa como quantitativamente, em relação aos pesquisadores não bolsistas do CNPq.

No início dos anos 90, o perfil médio aproximado dos pesquisadores de produtividade na escala de graduação IIC, IIB, IIA, IC, IB e IA exibia além dos qualificativos de maturidade, liderança e orientação, uma produção típica de 1, 3, 5, 7, 10 e 15 artigos publicados, respectivamente, nos últimos cinco anos ${ }^{48}$. A título de ilustração, neste início de milênio, essas médias evoluíram para 11, 14, 19, 24, 35 e 50 artigos, respectivamente, nas mesmas categorias ${ }^{48}$. Outro dado interessante é que, atualmente, o topo da produtividade para pesquisadores do nível I é atingida em idades superiores a 50 anos, registrando-se a manutenção desse patamar de excelência até os 60 anos, com pequeno declínio até os $70 \operatorname{anos}^{48}$. Tal perfil é extremamente curioso, pois não é normalmente verificado em qualquer outro tipo de atividade humana, refletindo de forma dramática o impacto da bolsa de produtividade na manutenção do elevado perfil intelectual e acadêmico dos pesquisadores.

Entretanto, na área de Química Inorgânica, os dados apresentados na Tabela 6 devem ser olhados com preocupação. Em primeiro lugar, a redução do número de bolsistas é bastante acentuada (de 66 bolsistas em 1990 para 48 em 2001), com o agravante que a maior queda foi observada no nivel IIC (de 35 para 9 bolsistas). Segundo as ponderações já feitas, isso pode estar sinalizando uma queda acentuada no potencial de renovação competitivo da área de Química Inorgânica. Pela lógica, com menos ingressantes no sistema de bolsas, a tendência futura seguramente será de queda. Esse quadro é ainda mais sério, se considerarmos que nesse cenário também estão incluídos os pesquisadores de sub-áreas correlatas, como de materiais e catálise, que estão lotados em Departamentos de Química Inorgânica. Portanto, o decréscimo constatado na população de bolsistas inorgânicos na última década não pode ser atribuído à migração dos pesquisadores para áreas correlatas.

\section{PROJEÇÕES FUTURAS E RECOMENDAÇÕES}

Os dados apresentados permitem compreender um pouco melhor a química inorgânica brasileira. Esses dados devem ser compa- rados com os apresentados pelas demais áreas, neste e nos trabalhos deste volume.

Os indicadores demonstram que a Inorgânica, mesmo incorporando atividades nas sub-áreas correlatas, ainda permanece como sendo a de menor dimensão na Química Brasileira. Apesar de pequena, entretanto, deve ser enfatizado que o perfil levantado, descreve uma área pujante, bastante diversificada e renovada, com intensa atividade de pesquisa e riqueza temática, em níveis comparáveis aos dos países desenvolvidos.

Considerando as linhas temáticas em investigação, a ampla variedade existente é comprometida apenas pelo pequeno número de grupos atuantes nas Universidades brasileiras. Assim, ao lado de um estímulo generalizado, devem ser reforçadas algumas especialidades estratégicas ou de maior interesse para o país, como a química organometálica, catálise, bioinorgânica e química supramolecular.

Uma especial atenção deveria ser dirigida para o surgimento de uma nova tendência, representada pela nanotecnologia molecular. Esse enfoque é particularmente atraente na área de inorgânica, pela sua abrangência e implicações. Sob muitos aspectos, a nanotecnologia molecular pode ser vista como uma extensão da química supramolecular, à medida que novas arquiteturas moleculares funcionais começam a ser projetadas visando uma funcionalidade específica, como um dispositivo capaz de realizar uma tarefa, ou ainda buscando a mimetização de sistemas biológicos. Esse enfoque tem perspectivas ilimitadas em bioinorgânica, na área de materiais e em catálise. Note-se que o emprego de catalisadores nanoparticulados ou partículas impregnadas poderá abrir novas perspectivas, interpondo-se entre a catálise homogênea e a catálise heterogênea, suscitando uma gama de desafios, em termos das teorias cinéticas e mecanísticas que terão que ser desenvolvidas.

O baixo nível de contratação de docentes nas universidades e a dinamização das linhas de pesquisas dos grupos consolidados, que passam a migrar rapidamente para novos interesses, podem trazer como consequiência o abandono ou declínio de linhas já estabelecidas. Isso tende a se agravar no futuro, representando uma ameaça aos investimentos já feitos em ciência e tecnologia. Assim, o papel de renovação, exercido pela contratação de jovens docentes precisa ser seriamente considerado e reforçado, particularmente na área de Química Inorgânica, onde a maior parte dos pesquisadores de nível I já conta com o tempo exigido para a aposentadoria. Além da contratação de novos docentes pelas Universidades, as agências de financiamento precisam garantir a sustentabilidade dos mesmos, para que pos-

Tabela 6. Distribuição dos Bolsistas de Produtividade do CNPq (número absoluto, por área, e o percentual correspondente) ${ }^{48}$

\begin{tabular}{|c|c|c|c|c|c|c|c|c|c|c|c|}
\hline ANO & TIPO & QI & $\%$ & QA & $\%$ & QO & $\%$ & FQ & $\%$ & TOTAL & $\%$ \\
\hline \multirow[t]{7}{*}{1990} & IA & 9 & 2,7 & 3 & 0,9 & 17 & 5,1 & 9 & 2,7 & 38 & 11,4 \\
\hline & IB & 3 & 0,9 & 2 & 0,6 & 15 & 4,5 & 11 & 3,3 & 31 & 9,3 \\
\hline & IC & 2 & 0,6 & 7 & 2,1 & 17 & 5,1 & 14 & 4,2 & 40 & 12,0 \\
\hline & IIA & 6 & 1,8 & 7 & 2,1 & 19 & 5,7 & 10 & 3,0 & 42 & 12,6 \\
\hline & IIB & 11 & 3,3 & 8 & 2,4 & 25 & 7,5 & 18 & 5,4 & 62 & 18,6 \\
\hline & IIC & 35 & 10,5 & 16 & 4,8 & 48 & 14,4 & 21 & 6,3 & 120 & 36,0 \\
\hline & Total & 66 & 19,8 & 43 & 12,9 & 141 & 42,3 & 83 & 24,9 & 333 & 100 \\
\hline \multirow[t]{7}{*}{2001} & IA & 10 & 2,9 & 7 & 2,0 & 10 & 2,9 & 16 & 4,6 & 43 & 12,3 \\
\hline & IB & 4 & 1,1 & 2 & 0,6 & 11 & 3,2 & 12 & 3,4 & 29 & 8,3 \\
\hline & IC & 9 & 2,6 & 10 & 2,9 & 14 & 4,0 & 18 & 5,2 & 51 & 14,6 \\
\hline & IIA & 5 & 1,4 & 12 & 3,4 & 22 & 6,3 & 23 & 6,6 & 62 & 17,8 \\
\hline & IIB & 11 & 3,2 & 25 & 7,2 & 32 & 9,2 & 22 & 6,3 & 90 & 25,8 \\
\hline & IIC & 9 & 2,6 & 18 & 5,2 & 27 & 7,7 & 20 & 5,7 & 74 & 21,2 \\
\hline & Total & 48 & 13,8 & 74 & 21,2 & 116 & 33,2 & 111 & 31,8 & 349 & 100 \\
\hline
\end{tabular}

QA = Química Analítica, QI = Química Inorgânica, QO = Química Orgânica e FQ = Físico-Química, 
sam atingir mais rapidamente o nível de maturidade exigido para a sobrevivência no meio científico nacional e internacional.

Os índices de atividade, em termos comparativos, indicam portanto a necessidade de um esforço programado e concentrado na área de Química Inorgânica, que leve à formação de maior número de pesquisadores competitivos, capazes de repor, sustentar e, ainda, de promover a renovação dos recursos humanos e da própria ciência, sem perda de qualidade. Recomenda-se à CAPES e ao CNPq, assim como às demais Agências, um sólido esforço nesse sentido, assegurando as bolsas necessárias para a manutenção dos programas de pós-graduação em Química Inorgânica, até que a área recupere o patamar de atuação, em termos comparativos, observado nas décadas passadas.

\section{AGRADECIMENTOS}

Os autores agradecem à $\mathrm{J}$. Gomes pela pesquisa inicial no Webofscience-ISI, ao C. R. Neri, pela apresentação dos resultados, e ao Prof. A. A. S. da Gama pela ajuda e informações complementares.

\section{REFERÊNCIAS}

1. Filgueiras, C. A.; Quim. Nova 1979, 2, 29.

2. Rocha Filho, R. C.; Biaggio, S. R.; Quim. Nova 1984, 7, 85

3. Rocha Filho, R. C.; $S B Q$ 10, Edição Comemorativa 1987, 30.

4. Rocha Filho, R. C.; Biaggio, S. R.; Bocchi, N.; Quim. Nova 1997, 20 (Especial), 67.

5. Massabni, A. C.; Quim. Nova 1982, 5, 143.

6. Felcman, J.; Mangrich, A. S.; da Cruz, G. M.; Tsunoda, M.; Quim. Nova 1989, 12, 379.

7. Ferreira, A. M. D.; Serra, O. A.; Quim. Nova 1995, 18, 505.

8. Seidl, P.; da Fonseca, A. F. M.; Gomes, H. E.; Lima, I. M. C.; Quim. Nova 1979, 2, 91.

9. Craveiro, A. A.; Quim. Nova 1985, 8, 121.

10. Cagnin, M. A. H.; Quim. Nova 1987, 10, 1987.

11. de Oliveira, A. B.; Paniago, E.; Cagnin, M. A. H.; Quim. Nova 1985, 8, 46.

12. Cagnin, M. A. H.; Quim. Nova 1983, 6, 156.

13. Ihde, A. J.; The Development of Modern Chemistry, Harper \& Row: New York, 1966.

14. Pauling, L.; The Nature of the Chemical Bond, Cornell Univ. Press: Ithaca, 1940.

15. Hainberger, L.; Quim. Nova 1983, 6, 55.

16. Giesbrecht, E.; Quim. Nova 1981, 4, 96.

17. Giesbrecht, E.; Quim. Nova 1985, 8, 275.

18. Chagas, A. P.; Toma, H. E.; Quim. Nova 1991, 14, 149; Toma, H. E.; Quim. Nova 1996, 19, 578.

19. Buyers, A.G.; Giesbrecht, E.; Audrieth, L.F.; J. Inorg. Nucl. Chem. 1957, $5,133$.

20. Vichi, E. J. S.; Quim. Nova 1983, 6, 152.

21. Krumholz, P.; Bril, K. J.; Fr. Pat. 1,207.954 1960

22. Krumholz, P.; Spectrochim. Acta 1958, 10, 269.

23. Barreto, L. A.; Prescott, E.; Quim. Nova 1997, 20 (Especial), 15.

24. Paniago, E. B.; Quim. Nova 1997, 20 (Especial), 23.

25. Teixeira, J. M.; Noções de Química Inorgânica, Ed. Francisco Alves: RJ, 1878.

26. Ostwald, W.; Grundlinien der Anorganischen Chemie, Verlag: Dresden, 1919.

27. Riesenfeld, E. H.; Practicas de Química Inorgânica, tradução 2a. ed., Ed. Labor: Barcelona, 1943 (1a. ed., 1928).

28. Oppenheimer, C.; Grundriss der Anorganischen Chemie, Verlag: Leipzig, 1920.

29. Hofmann, K. A.; Lehrbuch der Anorganischen Chemie, Druck e Verlag: Brauschweig, 1924.

30. Emeléus, H. J.; Anderson, J. S.; Modern Aspects of Inorganic Chemistry, Routledge \& Kegan: London, 1938.

31. Wells, A. F.; Structural Inorganic Chemistry, Oxford Univ. Press: London, 1945.

32. Sidgwick, N. V.; The Chemical Elements and Their Compounds, Oxford Univ. Press: London, 1950.

33. Moeller, T.; Inorganic Chemistry, John Wiley \& Sons: New York, 1952.

34. Gilreach, E. S.; Fundamental Concepts of Inorganic Chemistry, McGrawHill: New York, 1958.

35. Kleinberg, J.; Argersinger Jr., W. J.; Griswold, E.; Inorganic Chemistry, D. C. Heath: Boston, 1960.

36. Cotton, F. A.; Wilkinson, G.; Advanced Inorganic Chemistry, Interscience Publ.: New York, 1962

37. Phillips, C. S. G.; Williams, R. J. P.; Inorganic Chemistry, Oxford Univ. Press: Oxford, 1965.

38. Gilreach, E. S.; Fundamental Concepts of Inorganic Chemistry, McGrawHill: New York, 1958.

39. Jolly, W. L.; A Química dos Não Metais, trad., Ed. Edgard Blücher/EDUSP: São Paulo, 1966.

40. Cotton, F. A., Chemical Applications of Group Theory, Wiley-Interscience: New York, 1963.

41. Ohlweiler, O. A.; Química Inorgânica, Ed. Edgard Blücher/EDUSP: São Paulo, 1971.

42. Toma, H. E.; Química Bioinorgânica, Ed. OEA: Washington DC, 1984.

43. Barros, H. L. C.; Química Inorgânica, Uma Introdução, Ed. UFMG: Belo Horizonte, 1992

44. Cagnin, M. A. H.; Quim. Nova 1981, 4, 49.

45. Cagnin, M. A. H.; Quim. Nova 1988, 11, 478.

46. da Gama, A. A. S.; Nome, F. J.; Machado, J. C.; Quim. Nova 1999, 22, 443.

47. da Gama, A. A. S.; comunicação pessoal.

48. Toma, H. E.; Memórias de reuniões do CA-CNPq de 1990, 2001 e 2002.

\section{APÊNDICE}

\section{DIVISÃO DE QUÍMICA INORGÂNICA}

Número de filiados: 257 (atual)

1994/1996

DIRETOR:

VICE-DIRETOR:

TESOUREIRO:

$1996 / 1998$

DIRETOR:

VICE-DIRETOR:

TESOUREIRO:

$1998 / 2000$

DIRETOR:

VICE-DIRETOR:

TESOUREIRO:

2000/2002

DIRETOR:

VICE-DIRETOR:

TESOUREIRO:
Eduardo J. S. Vichi

Yoshitaka Gushikem

Alzir Azevedo Batista

Carlos Alberto L. Filgueiras

Manfredo Horner

Alzir Azevedo Batista

Alzir Azevedo Batista

Ícaro de Souza Moreira

Roberto de Barros Faria

Heloisa de O. Beraldo

Annelise E. Gerbase

Jaisa F. Soares 